\title{
Mobility Calculating of Planar Mechanisms in a New Method
}

\author{
Yuwang Liu \\ Sciences \\ Shenyang, China \\ liuyuwang@sia.cn \\ Chen Chen* \\ Northeastern University \\ Shenyang, China \\ chenchen1195@gmail.com \\ * Corresponding author
}

Shenyang Institute of Automation Chinese Academy of

\author{
Tian Tian \\ Shenyang Ligong University \\ Shenyang, China \\ tian309114@163.com \\ Yingbo Lei \\ Shenyang Ligong University \\ Shenyang, China \\ linjiangtingyu@163.com
}

\begin{abstract}
In this article, a new method of calculating mobility for planar kinematic chains, based on kinematic graph of mechanism and matrix theory, is put forward. We proposed a new conception of network graph of mechanism to describe kinematic chains instead of kinematic graph of mechanism. With a network graph of mechanism, the displacements of components produced by applied forces can be calculated effectively and simply. Futhermore, matrixes can be established by displacement and force equation groups. By means of analyzing the matrixes, we will obtain the mobility of a mechanism with no efforts as the mobility equals to the dimension of the matrix arrived at from equation groups. In addition, this new method is applied to various kinds of mechanisms in plane efficiently and correctly including the kinematic chains with passive constraints and common constraints. The method of this paper can be extended to calculate mobility of spatial mechanisms too.
\end{abstract}

Keywords-mobility calculating; network graph of mechanism; matrix analysis; displacement; planar machanism

\section{INTRODUCTION}

The study on mobility calculation has been continued for more than 100 years. Various methods of mobility calculation have been put forward, and many methods were analyzed in [1] by Gogu. As early as the19th century, Reuleaux [2] defined mechanism firstly and made a system study on kinematic pairs. And on this basis Grübler [3] puts forward the general formula of degree of freedom for simple planar chains. Crossley [4] analyzed planar link mechanisms based on Grübler's formula, and came up with the relation between kinematic pairs and the number of bodies. But for the link mechanisms with four joints, the calculation of link number should use the method of trial and error which might make mistakes. Bagci [5] presented a general formula of mobility calculation for mechanisms of $n$ links and $k$ loops. The formula takes into consideration that passive constraint, common constraint and passive degrees of freedom, but its results are not correct for some mechanisms. Traditionally, the classical formula of Chebyshev-Grübler [6] is used to calculate planar mechanisms' mobility. Obviously, the formula is convenient to obtain mobility of some simple link mechanisms. But for mechanisms of passive constraints, common constraints or passive degrees of freedom, it is not easy to judge and even gets wrong results.

Recently, further researches have been reached into the mobility of rigid mechanisms [7, 8], metamorphic mechanisms [9-12] based on screw theory which is difficult to understand. The methods using Lie group [13], Lie algebra [14] and linear transformation [15] are also complicated. Dongchao Yang came up with a simple method of mobility calculation with Jacobian [16], but the rank of coefficient matrix must be calculated by MATLAB.

A new method would be presented for mobility calculation of planar mechanisms in this paper. This method makes a new way to show the kinematic chains instead of kinematic graph of mechanism. In this case, mobility calculation turns to determine the dimension of a matrix which is brought by some simple equation groups as the dimension of the matrix equals to the mobility of mechanism.

\section{NEW THEORY OF MOBILITY CALCULATION FOR PLANAR MECHANISMS}

\section{A. Conception and principles of network graph of mechanism}

Kinematic graph of mechanism is a simple sketch that expresses the movement of mechanism kinematics precisely using simple lines and signs. However, the positions of components in kinematic graph need to be determined accurately and proportionally. The method of this paper only requires linking relation of components, thus a new conception of network graph of mechanism is presented for describing kinematic chains instead of kinematic graph of mechanisms, which applies to this method appropriately and simply. The details of principles of network graph of mechanism are listed as follows. 
Network graph of mechanism includes elasticity links, nodes and the root. The elastic links and nodes denote bodies and pairs in kinematic chains respectively. The size of components of network graph of mechanism need not de proportional to the actual components as it has absolutely no effect on mobility.

If the bodies link to the root in kinematic graph of mechanism, the nodes between them will be canceled in network graph.

If the bodies whose joints are single in open or mixed kinematic chains, they will get two nodes in network graph of mechanism.

The relative position of two elastic links in network graph of mechanism should be horizontal, vertical or at a 45-degree angle when they linked by one node, so as to analyze simply.

\section{B. Establish equation groups based on network graph of mechanism}

For the four-bar linkage in Fig .1a, the following will present its transformation from kinematic graph to network graph of mechanism and the process of establishing equation groups.

Based on the principles above, the horizontal line AD in Fig .1b denotes the root of the four-bar linkage; the joints $\mathrm{B}$ and $\mathrm{C}$ are denoted as $m_{1}$ and $m_{2}$; the nodes $\mathrm{A}$ and $\mathrm{D}$ are canceled as the bodies 1 and 3 link to the root directly; the elastic links $L_{1}, L_{2}, L_{3}$ denote the links 1, 2, 3 respectively.

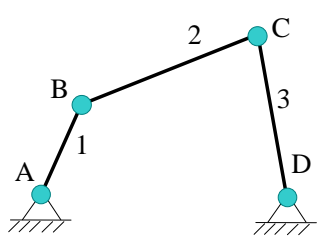

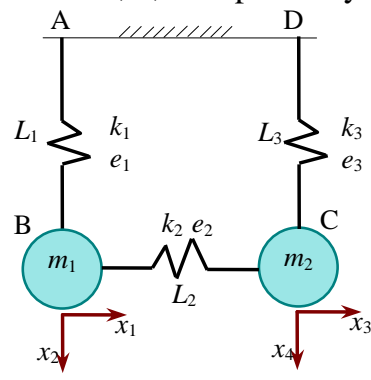

b
Figure 1. a) Four-bar linkage. b) Network graph of four-bar linkage.

We denote supposed forces applied on nodes by $f_{2 j-1}$ and $f_{2 j}(j=1,2,3 \ldots n)$. Similarly, the associated horizontal and vertical displacements of $m_{j}$ produced by the forces will be denoted by $x_{2 j-1}$ and $x_{2 j}$ respectively. $L_{i}(i=1,2,3 \ldots n)$ denotes the unstretched length of the $i$ th elastic link whose stiffness is $k_{i}$, and the corresponding elongation is $e_{i}$. Thus, we can easy to get the description of the four-bar linkage in Fig. $1 b$.

According to the deformation of elastic links, the displacement of node $m_{1}$ in vertical direction is [17]

$$
e_{1}=\sqrt{x_{1}^{2}+\left(x_{2}+L_{1}\right)^{2}}-L_{1}
$$

In order to simplify the formula, the Taylor expansion is used to make the elongation of the elastic link has a linear relationship with the end displacement. Naturally, there is

$$
\begin{aligned}
e_{1}= & \sqrt{x_{1}^{2}+\left(x_{2}+L_{1}\right)^{2}}-L_{1} \\
= & \sqrt{x_{1}^{2}+x_{2}^{2}+2 x_{2} L_{1}+L_{1}^{2}}-L_{1} \\
= & L_{1} \sqrt{1+\left(x_{1}^{2}+x_{2}^{2}\right) / L_{1}^{2}+2 x_{2} / L_{1}+L_{1}^{2}}-L_{1} \\
= & L_{1}+\left(x_{1}^{2}+x_{2}^{2}\right) / 2 L_{1}+x_{2}+ \\
& L_{1} O\left(\left(\left(x_{1}^{2}+x_{2}^{2}\right) / L_{1}^{2}+2 x_{2} / L_{1}\right)^{2}\right)-L_{1} \\
= & x_{2}+\left(x_{1}^{2}+x_{2}^{2}\right) / 2 L_{1}+ \\
& L_{1} O\left(\left(\left(x_{1}^{2}+x_{2}^{2}\right) / L_{1}^{2}+2 x_{2} / L_{1}\right)^{2}\right)
\end{aligned}
$$

Where $O\left(\left(\left(x_{1}^{2}+x_{2}^{2}\right) / L_{1}^{2}+2 x_{2} / L_{1}\right)^{2}\right)$ is the Peano remainder.

Obviously, $\left(x_{1}^{2}+x_{2}^{2}\right) / 2 L_{1}$ is small compared to $x_{2}$, and the Peano remainder is even smaller. Thus, all the terms above except the first one can be neglected and approximately arrive at

$$
e_{1}=x_{2}
$$

The argument of the first elastic link indicates that the elongation of elastic link is its displacement along its initial direction approximately. Similarly, there are

$$
e_{2}=x_{3}-x_{1}, \quad e_{3}=x_{4}
$$

These three displacement equation groups can be encoded in

$$
e=\mathbf{A} x \text { where } \mathbf{A}=\left[\begin{array}{cccc}
0 & 1 & 0 & 0 \\
-1 & 0 & 1 & 0 \\
0 & 0 & 0 & 1
\end{array}\right]
$$

The matrix $\mathbf{A}$ is called displacement matrix here.

There is no doubt that the elastic links are in the range of flexibility, so Hooke's Law (if the material is in the range of flexibility, its deformation and the force loading on it will be proportional) applies to them. Naturally

$$
y_{\mathrm{i}}=k_{i} e_{i}
$$

Or, in matrix terms

$$
y=\mathbf{K} e \quad \text { where } \quad \mathbf{K}=\left[\begin{array}{ccc}
k_{1} & 0 & 0 \\
0 & k_{2} & 0 \\
0 & 0 & k_{3}
\end{array}\right]
$$

The matrix $\mathbf{K}$ is called stiffness matrix here.

According to the force equilibrium on each node in horizontal and vertical direction, the equilibrium equation groups can be arrived at 


$$
y_{2}-f_{1}=0, y_{1}-f_{2}=0, y_{2}-f_{3}=0, y_{3}-f_{4}=0
$$

Or, in matrix terms

$$
\mathbf{B} y=f \text { where } \mathbf{B}=\left[\begin{array}{ccc}
0 & -1 & 0 \\
1 & 0 & 0 \\
0 & 1 & 0 \\
0 & 0 & 1
\end{array}\right] \text { and } \mathbf{f}=\left[\begin{array}{c}
f_{1} \\
f_{2} \\
f_{3} \\
f_{4}
\end{array}\right]
$$

The matrix $\mathrm{B}$ is called equilibrium matrix here.

The three matrixes above we arrived at can lead us to work out the mobility by matrix analysis.

\section{Work out mobility}

As is showed above, the relationship between A. and B can be recognized as $\mathbf{B}=\mathbf{A}^{\mathbf{T}}$. Gathering the previous steps

$$
e=\mathbf{A} x, y=\mathbf{K} e, \mathbf{B} y=f, \mathbf{B}=\mathbf{A}^{\mathbf{T}}
$$

We arrive at

$$
\mathbf{B} y=\mathbf{A}^{\mathbf{T}} y=\mathbf{A}^{\mathbf{T}} \mathbf{K} e=\mathbf{A}^{\mathbf{T}} \mathbf{K} \mathbf{A} x=\mathbf{f}
$$

Namely,

$$
\mathbf{S}=\mathbf{A}^{\mathbf{T}} \mathbf{K A}
$$

Where

$$
\begin{aligned}
& \mathbf{S}=\mathbf{A}^{\mathbf{T}} \mathbf{K A} \\
& =\left[\begin{array}{ccc}
0 & -1 & 0 \\
1 & 0 & 0 \\
0 & 1 & 0 \\
0 & 0 & 1
\end{array}\right]\left[\begin{array}{ccc}
k_{1} & 0 & 0 \\
0 & k_{2} & 0 \\
0 & 0 & k_{3}
\end{array}\right]\left[\begin{array}{cccc}
0 & 1 & 0 & 0 \\
-1 & 0 & 1 & 0 \\
0 & 0 & 0 & 1
\end{array}\right] \\
& =\left[\begin{array}{cccc}
k_{2} & 0 & -k_{2} & 0 \\
0 & k_{1} & 0 & 0 \\
-k_{2} & 0 & k_{2} & 0 \\
0 & 0 & 0 & k_{3}
\end{array}\right]
\end{aligned}
$$

After elementary row transformation, the matrix is

$$
\mathbf{S}^{\prime}=\left[\begin{array}{cccc}
k_{2} & 0 & -k_{2} & 0 \\
0 & k_{1} & 0 & 0 \\
0 & 0 & 0 & k_{3} \\
0 & 0 & 0 & 0
\end{array}\right]
$$

The rank of matrix $\mathrm{S}$ is 3 , and the dimension of null space of matrix $\mathrm{S}$ is $n-r(\mathbf{S})=1$. According to Chebyshev-Gruebler's equation for planar chains, we arrive at

$$
\mathrm{DOF}=3(N-1)-2 P_{l}=3 \times(4-1)-2 \times 4=1
$$

Thus, there is

$$
\mathrm{DOF}=\operatorname{Dim} .\{\mathbf{S}\}
$$

$\operatorname{Dim} .\{\mathbf{S}\}$ is the dimension of null space of matrix $\mathrm{S}$.

This result means that the dimension of null space of matrix $\mathrm{S}$ equals to its respective mobility of mechanism. It has similar result in [16] that dimension of fundamental solutions of homogeneous equation group equals to the number of active joints.

\section{PROOF OF NEW THEORY BY EXAMPLES}

The proof of closed kinematic chains is showed in Fig .1 which takes a four-bar linkage as an example. Undermentions are proof of Open kinematic chains and mixed kinematic chains.

\section{A. Open kinematic chains}

Fig .2 shows a three-bar linkage of open kinematic chain and its corresponding network graph of mechanism.
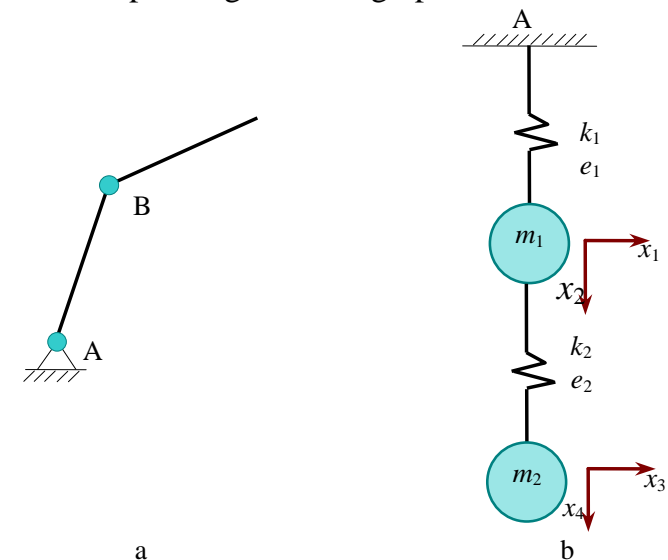

Figure 2. a) Three-bar linkage of open kinematic chain. b) Network graph of three-bar linkage.

As is showed in previous section, the displacement equation groups can be easily arrived at

$$
e_{1}=x_{2}, e_{2}=x_{4}-x_{2}
$$

In matrix terms, the displacement matrix is

$$
\mathbf{A}=\left[\begin{array}{cccc}
0 & 1 & 0 & 0 \\
0 & -1 & 0 & 1
\end{array}\right]
$$

Similarly, the stiffness matrix

$$
\mathbf{K}=\left[\begin{array}{cc}
k_{1} & 0 \\
0 & k_{2}
\end{array}\right]
$$

The equilibrium equation groups are

$$
f_{1}=f_{3}=0, f_{2}=y_{1}-y_{2}, f_{4}=y_{2}
$$


In matrix terms, the equilibrium matrix is

$$
\mathbf{B}=\left[\begin{array}{ll}
0 & 0 \\
1 & -1 \\
0 & 0 \\
0 & 1
\end{array}\right]
$$

Equations (18), (19) and (21) bring

$$
\begin{aligned}
\mathbf{S} & =\mathbf{A}^{\mathrm{T}} \mathbf{K A} \\
& =\left[\begin{array}{cc}
0 & 0 \\
1 & -1 \\
0 & 0 \\
0 & 1
\end{array}\right]\left[\begin{array}{cc}
k_{1} & 0 \\
0 & k_{2}
\end{array}\right]\left[\begin{array}{cccc}
0 & 1 & 0 & 0 \\
0 & -1 & 0 & 1
\end{array}\right] \\
& =\left[\begin{array}{cccc}
0 & 0 & 0 & 0 \\
0 & k_{1}+k_{2} & 0 & -k_{2} \\
0 & 0 & 0 & 0 \\
0 & -k_{2} & 0 & k_{2}
\end{array}\right]
\end{aligned}
$$

As the rank of matrix $S$ is 2 with inspection, the dimension of null space of matrix $\mathrm{S}$ is 2 . According to Chebyshev-Gruebler's equation, we arrive at $\mathrm{DOF}=2$.

As expected, the result is the same as (16).

A five-bar linkage of mixed kinematic chain and its corresponding network graph of mechanism is showed in Fig .3a and $b$.

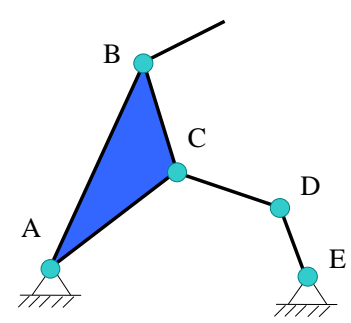

a

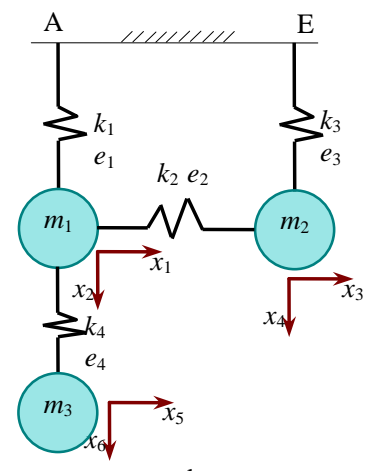

b
Figure 3. a) Five-bar linkage of mixed kinematic chain. b) Network graph of five-bar linkage.

Similarly, the displacement matrix, stiffness matrix and equilibrium matrix can be derived easily based on the steps in previous section. Then we can arrive at

$$
\begin{aligned}
& \mathbf{S}=\mathbf{A}^{\mathrm{T}} \mathbf{K A} \\
& =\left[\begin{array}{cccc}
0 & -1 & 0 & 0 \\
1 & 0 & 0 & -1 \\
0 & 1 & 0 & 0 \\
0 & 0 & 1 & 0 \\
0 & 0 & 0 & 0 \\
0 & 0 & 0 & 1
\end{array}\right]\left[\begin{array}{cccc}
k_{1} & 0 & 0 & 0 \\
0 & k_{2} & 0 & 0 \\
0 & 0 & k_{3} & 0 \\
0 & 0 & 0 & k_{4}
\end{array}\right] \\
& {\left[\begin{array}{cccccc}
0 & 1 & 0 & 0 & 0 & 0 \\
-1 & 0 & 1 & 0 & 0 & 0 \\
0 & 0 & 0 & 1 & 0 & 0 \\
0 & -1 & 0 & 0 & 0 & 1
\end{array}\right]} \\
& =\left[\begin{array}{cccccc}
k_{2} & 0 & -k_{2} & 0 & 0 & 0 \\
0 & k_{1}+k_{4} & 0 & 0 & 0 & -k_{4} \\
-k_{2} & 0 & k_{2} & 0 & 0 & 0 \\
0 & 0 & 0 & k_{3} & 0 & 0 \\
0 & 0 & 0 & 0 & 0 & 0 \\
0 & -k_{4} & 0 & 0 & 0 & k_{4}
\end{array}\right]
\end{aligned}
$$

By making an elementary row transformation on $\mathbf{S}$, the matrix is derived as

$$
\mathbf{S}^{\prime}=\left[\begin{array}{cccccc}
k_{2} & 0 & -k_{2} & 0 & 0 & 0 \\
0 & k_{1} & 0 & 0 & 0 & 0 \\
0 & -k_{4} & 0 & 0 & 0 & k_{4} \\
0 & 0 & 0 & k_{3} & 0 & 0 \\
0 & 0 & 0 & 0 & 0 & 0 \\
0 & 0 & 0 & 0 & 0 & 0
\end{array}\right]
$$

The rank of matrix $\mathbf{S}$ is 4 , so the dimension of null space of matrix $S$ is 2 . The mobility of the mixed six-bar linkage is 2. Thus, the result is the same as (16).

\section{APPLICATIONS OF NEW THEORY ON SPECIAL KINEMATIC CHAINS}

\section{A. Mobility calculation of mechanisms with passive constraints}

This method is applied to mechanisms with passive constraints appropriately, as it is no need to take them into consideration. That is to say, the mobility can be calculated directly without removing passive constraints.

Just like the kinematic chain in Fig .4a, its method of transformation into network graph in Fig. $4 \mathrm{~b}$ is similar to previous mechanisms.

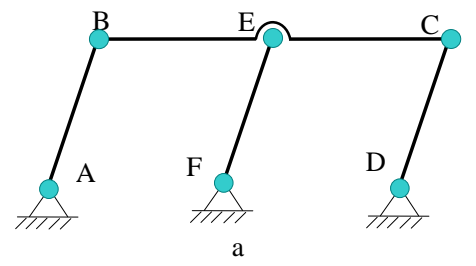




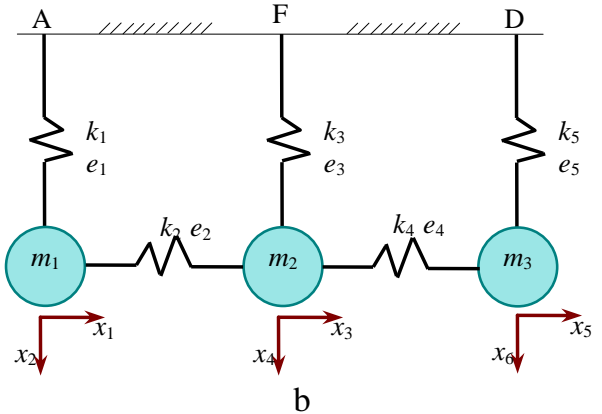

Figure 4. a) Mechanism with passive constraint. b) Network graph of mechanism with passive constraint.

After analyzing the network graph of mechanism in Fig .4b, it brings

$$
\begin{aligned}
& \mathbf{S}=\mathbf{A}^{\mathbf{T}} \mathbf{K A} \\
& =\left[\begin{array}{ccccc}
0 & -1 & 0 & 0 & 0 \\
1 & 0 & 0 & 0 & 0 \\
0 & 1 & 0 & -1 & 0 \\
0 & 0 & 1 & 0 & 0 \\
0 & 0 & 0 & 1 & 0 \\
0 & 0 & 0 & 0 & 1
\end{array}\right]\left[\begin{array}{ccccc}
k_{1} & 0 & 0 & 0 & 0 \\
0 & k_{2} & 0 & 0 & 0 \\
0 & 0 & k_{3} & 0 & 0 \\
0 & 0 & 0 & k_{4} & 0 \\
0 & 0 & 0 & 0 & k_{5}
\end{array}\right] \\
& {\left[\begin{array}{cccccc}
0 & 1 & 0 & 0 & 0 & 0 \\
-1 & 0 & 1 & 0 & 0 & 0 \\
0 & 0 & 0 & 1 & 0 & 0 \\
0 & 0 & -1 & 0 & 1 & 0 \\
0 & 0 & 0 & 0 & 0 & 1
\end{array}\right]} \\
& =\left[\begin{array}{cccccc}
k_{2} & 0 & -k_{2} & 0 & 0 & 0 \\
0 & k_{1} & 0 & 0 & 0 & 0 \\
-k_{2} & 0 & k_{2}+k_{4} & 0 & -k_{4} & 0 \\
0 & 0 & 0 & k_{3} & 0 & 0 \\
0 & 0 & -k_{4} & 0 & k_{4} & 0 \\
0 & 0 & 0 & 0 & 0 & k_{5}
\end{array}\right]
\end{aligned}
$$

By making an elementary row transformation on $\mathbf{S}$, the matrix is

$$
\mathbf{S}^{\prime}=\left[\begin{array}{cccccc}
k_{2} & 0 & -k_{2} & 0 & 0 & 0 \\
0 & k_{1} & 0 & 0 & 0 & 0 \\
0 & 0 & -k_{4} & 0 & k_{4} & 0 \\
0 & 0 & 0 & k_{3} & 0 & 0 \\
0 & 0 & 0 & 0 & 0 & k_{5} \\
0 & 0 & 0 & 0 & 0 & 0
\end{array}\right]
$$

The rank of matrix $\mathrm{S}$ is 5 , so the dimension of null space of matrix $\mathrm{S}$ is 1. Chebyshev-Gruebler's equation brings $\mathrm{DOF}=1$. So the result is the same as (16).

\section{B. Mobility calculation of mechanisms with common constraints}

This method is also applied to mechanisms with common constraints, but its principles of transformation from kinematic graph to network graph of mechanism are partly different. The differences are

The nodes in network graph of mechanism denote the bodies;

The elastic links denote the constraints between bodies or bodies and the root.

According to the principles, the transformation of the wedge mechanism in Fig .5a is showed in Fig .5b.

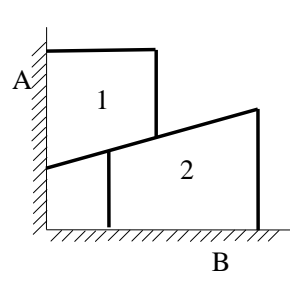

a

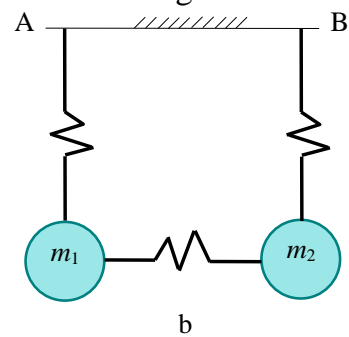

b
Figure 5. a) Wedge mechanism with common constraints. b) The network graph of wedge mechanism

Obviously, its network graph of mechanism has no different with that of the four-bar linkage in Fig .1b. So the mobility of this wedge mechanism with common constraints is single.

\section{Mobility calculation of mechanisms with higher pairs and passive degrees of freedom}

For mechanisms with higher pairs and passive degrees of freedom, the passive degrees of freedom need to be removed just like Chebyshev-Gruebler's method.

Fig .6a shows a mechanism with higher pairs and passive degrees of freedom. After removing its passive degrees of freedom, its principles of transformation into network graph of mechanism (Fig .6b) are similar to the mechanisms with common constraints.
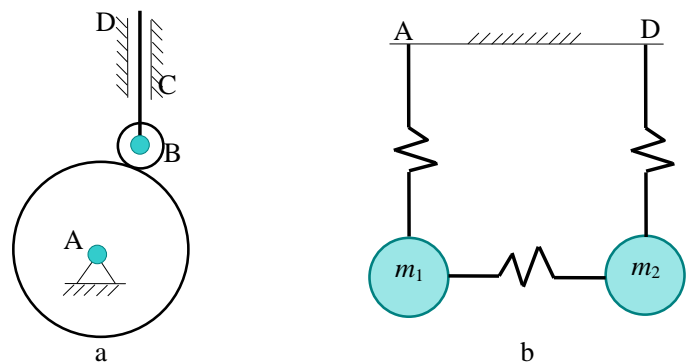

Figure 6. a) Mechanism with higher pairs and passive degrees of freedom. b) The network graph of the mechanism in a.

Obviously, the network graph of the mechanism in Fig .6b is the same as the four-bar linkage in Fig .1b after removing its passive degrees of freedom. Thus, we can get its mobility with no effort as its calculation is in previous section.

\section{CONCLUSIONS}

The above mentions approve that this new method applies to calculate the mobility of planar mechanisms simply. It is effective to analyze degrees-of-freedom of open kinematic chains, closed kinematic chains, mixed kinematic chains and some special kinematic chains with common constraints, passive constraints, etc. The crux of this method is the transformation from kinematic graph to network graph of mechanism which needs to be appropriate and correct. Besides, for some simple planar 
mechanisms, their mobility can be counted by inspection and for some kinematic chains whose network graph of mechanisms are identical have the same number of mobility.

\section{REFERENCES}

[1] Grigore Gogu, Mobility of mechanisms: A critical review, Mechanism and Machine Theory 40 (9) (2005) 1068-1097.

[2] F. Reuleaux, Theoretische Kinematic, Fridrich vieweg, Braunschweig, Germany, 1875 (English translation by A.B.W Kennedy, The kinematics of machinery, 1876, Reprinted Dover, 1963).

[3] M. Gruebler, Allgemeine Eigenschaften der zwanglaufigen ebenen inematischen Ketten Part I, Civilingenieur 29(1883) 167-200; Part II, Verh. Ver.Bef. Gew. 64 (1885) 179-223.

[4] F.R.E. Crossley, A contribution to Gruebler's theory in the number synthesis of planar mechanisms, J. Eng. Indust, ASME Trans, Series B 86 (1964) 1-8.

[5] C.Bagci, Degrees of freedom of motion in mechanisms, ASME J. Eng. Industry 93B (1971) 140-148.

[6] T.S. Mruthyunjaya, Kinematic structure of mechanisms revisited, Mechanism and Machine Theory 38 (2003) 279-320.

[7] Jing-Shan Zhao, Kai Zhou, Zhi-Jing Feng. Computation of the configuration degree of freedom of a spatial parallel mechanism by using reciprocal screw theory. Mechanism and Machine Theory 41 (2006) 1486-1504.

[8] Jing-Shan Zhao, Kai Zhou, Zhi-Jing Feng, A theory of degrees of freedom for mechanisms, Mechanism and Machine Theory 39 (6) (2004) 621-643.
[9] LI Shouzhong, YU Jingjun, and ZONG Guanghua. Type synthesis and principal freedom analysis of parallel flexure mechanisms based on screw theory, Chinese Journal of Mechanical Engineering 46 (13) (2010) 54-60.

[10] LI Duanling, ZHANG Zhonghai, DAI Jiansheng, Zhang Ketao, Overview and Prospects of Metamorphic Mechanism, Chinese Journal of Mechanical Engineering 46(13) (2010) 14-21.

[11] GAO Mingwang, ZHANG Xianmin, LIU Han. Experiment and Kinematic Design of 3-RRR Parallel Robot with High Speed[J]. ROBOT, 2013,35(6): 716-722.

[12] Maurizio Ruggiua, Xianwen Kong. Mobility and kinematic analysis of a parallel mechanism with both PPR and planar operation modes. Mechanism and Machine Theory 55 (2012) 7790.

[13] J.M. Rico, J. Gallardo, B. Ravani, Lie algebra and the mobility of kinematic chains, Journal of Robotic Systems 20 (8) (2003) 477 499

[14] Sameh Refaat, Jacques M. Herve, Saeid Nahavandi, Hieu Trinh, Asymmetrical three-DOFs rotational-translational parallelkinematics mechanisms based on Lie group theory, European Journal of Mechanics, A/Solids 25 (3) (2006) 550-558.

[15] Grigore Gogu, Mobility and spatiality of parallel robots revisited via theory of linear transformations, European Journal of Mechanics, A/Solids 24 (4) (2005) 690-711.

[16] Dong-Chao Yang, Jing Xiong, Xiang-Dong Yang, A simple method to calculate mobility with Jacobian. Mechanism and Machine Theory 43 (2008) 1175-1185.

[17] Steven J Cox, Matrix Analysis, CAAM 335,Sping 2012. 